\title{
Design and Implementation of Beidou/GPS Dual Mode Receiver
}

\author{
Li Zhou, a), Yijun Liu ${ }^{2}$, b) \\ ${ }^{1}$ School of Computer Science, Guangdong University of Technology China \\ ${ }^{2}$ School of Information Engineering, Guangdong University of Technology, Guangzhou 510006, China \\ a)876018232@qq.com \\ b)yjliu@gdut.edu.cn
}

\begin{abstract}
With the development of global satellite navigation systems, the design of multi-mode navigation receivers based on software radio has become a research hotspot in the entire navigation system. The design of a Beidou/GPS dualmode receiver based on the MAX2769B is introduced. The architecture of the FPGA+ARM is used to process satellite signals and finally achieve navigation positioning. It can be seen from the experiment that based on the Beidou/GPS dual-mode receiver, more observations are added on the basis of constellation optimization, which significantly reduces the positioning error and improves the positioning accuracy.
\end{abstract}

Key words: Beidou/GPS; FPGA+ARM; MAX2769B; global satellite navigation; constellation optimization.

\section{PREFACE}

Compared to the US GPS system, currently there are few satellites in the Beidou system space constellation. Therefore, the number of visible satellites at any observation point varies greatly with time, the geometrical distribution of satellites is uneven, and the GDOP value in the high latitudes and the western regions. Compared with the low latitudes and the eastern regions, and the Beidou GEO satellites are all above the equator, the latitude has a large correlation. Using GEO positioning alone will cause larger latitude errrs in positioning results [1]. Once GPS malfunctions or human interference occurs, it will seriously affect the normal operation of navigation and positioning or timing equipment. In order to achieve accurate and stable navigation and positioning functions, it is necessary to study how to integrate different satellite navigation and positioning system information and use GPS's mature space constellations to supplement the current defects of the Beidou space constellation [2]. The application market of the Beidou satellite navigation system has great potential. The development of a multi-mode receiver based on Beidou/GPS will have good prospects.

\section{OVERALL PLAN OVERVIEW}

Figure 1 shows the system design of Beidou/GPS dual-mode receiver, The Beidou/GPS signal reception process can be summarized in the following three steps:

Signal capture. The role of signal capture is to determine the Doppler shift and determination of a certain PRN satellite signal. The phase of the satellite ranging code is the sequential search used in this study.

Signal tracking. When the signal is present and capture is complete, the receiver enters this phase. In this process, a Delayed Locked Loop (DLL) is used to track the pseudo code phase, and a Phase Locked Loop (PLL) and a Frequency Locked Loop (FLL) are used to track carriers respectively. Phase and frequency. 


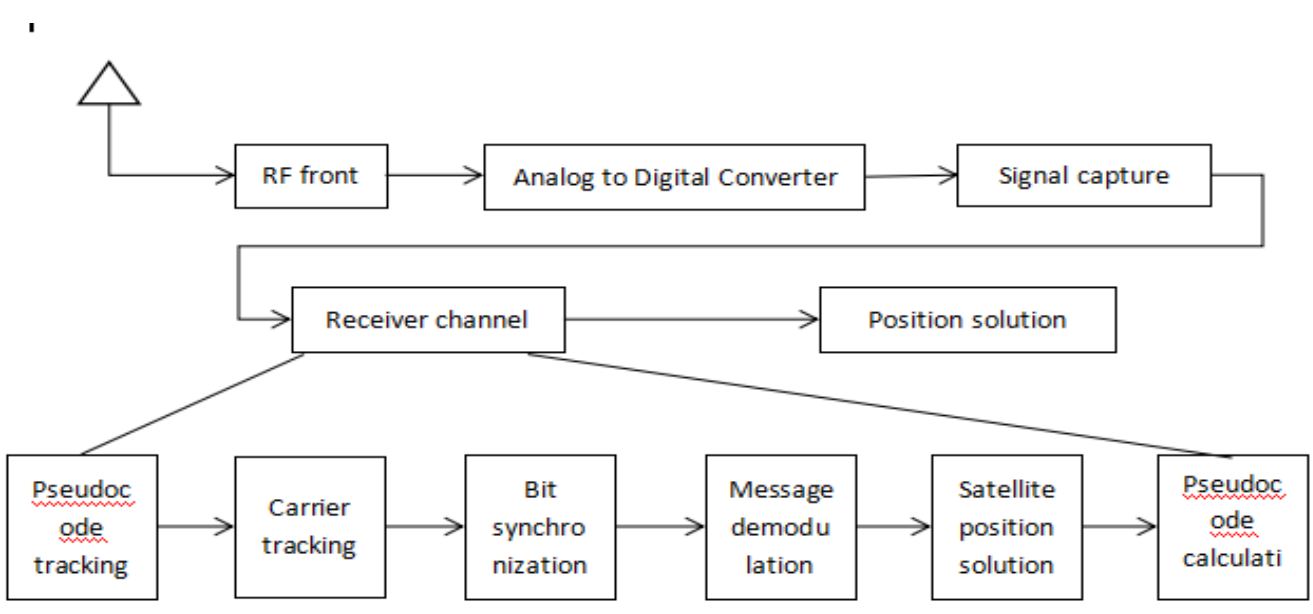

FIG.1. Overall design diagram

Text demodulation and location solution. After all the tracking loops are locked, the message can be demodulated, and then the position of the receiver can be obtained using the satellite position calculation and pseudo range positioning principles described in the previous section.

\section{BASEBAND SIGNAL PROCESSING}

The baseband processing module constituted by the FPGA, its main task is to implement the function of the correlator; the ARM function module is to uniformly deploy multiple channels of the entire receiver, and to manage signal processing processes such as signal acquisition, tracking, and synchronization. Finally, the settlement of the user receiver position is achieved and output. It is worth mentioning that the RF solution used in this study is the MAX2769, which converts the Beidou B1 frequency point $(1561.091 \mathrm{MHz})$ and GPS L1 frequency point $(1575.42 \mathrm{MHz})$ down to the intermediate frequency of $4.092 \mathrm{MHz}$ and obtains the digital intermediate frequency signal through the built-in ADC sampling. the FPGA is generally composed of several correlators. Since the RF hardware part provides the FPGA with Beidou and GPS digital IF signals including IF carrier signals, spreading codes (ie, pseudo-codes), and navigation messages, the baseband signal processing of the FPGA is generally performed. It consists of IF carrier stripping, pseudo code stripping, and correlators.

\section{IF Carrier Stripping}

The IF carriers are stripped, that is, the carrier and the information modulated on the carrier are demodulated. It consists of a Numerically Controlled Oscillator (NCO) and a multiplier. In order to peel off the carrier of the digital intermediate frequency and obtain the modulation code with modulated data, the carrier NCO will generate a replica carrier signal with the same frequency in phase, and then multiply the digital IF signal and shift the spectrum of the signal. The working process of the NCO can be described as that, driven by the system clock, the N-bit accumulators continuously accumulate counts, wherein the step is the phase control word $\mathrm{M}$. The phase accumulator is the core part of the entire NCO, and the essence is equivalent to performing the clock signal first. Divide the frequency, obtain the step signal by $M$ frequency again, and finally look up the output waveform value through the look-up table, each cycle outputs the positive/cosine wave of one cycle [3].

\section{ARM NAVIGATION SOLUTION MODULE IMPLEMENTATION}

\section{ARM Channel Control}

The microprocessor first initializes each channel. The initialization includes the allocation of satellites for each channel, the setting of the PRN code, the code NCO control word, and the carrier NCO control word. After the 
initialization is completed, each channel will search for and capture the satellites of the corresponding channel. During capture, the microprocessor will determine whether the baseband buffers new values (correlation values, carrier phase latch values, code phase latch values, and epochs). Parameters such as the count value), if there is a cache value, use the correlation value in the cache value to determine whether the satellite signal is captured. If the correlation value is too small, continue to adjust the channel parameter value until the signal is captured. After the capture is complete, the signal is tracked, the synchronization signal is then decoded, the navigation message is then decoded, and the position of the satellite is solved using the positional parameters in the navigation message. The pseudorange of the satellite from the receiver is calculated and then the minimum is passed. Multiplication solves the user's receiver position coordinates. Therefore, the ARM channel part is mainly divided into signal acquisition, signal tracking, signal synchronization, and navigation message resolution.

\section{Navigation Message Calculation}

After obtaining the local time of the receiver and the transmitting time of the satellite signal, the satellite pseudorange can be calculated first, and the space position of the satellite can be calculated by using the ephemeris parameters of the navigation message; the error can be corrected, and then the pseudorange and speed observation equation can be constructed to construct the observation. In the equation, the magnitude of the signal-to-noise ratio and the elevation angle of the satellite are used as the basis for the weights of the satellite observation equations. Finally, the observation equation is solved using the least squares method [4]. After solving, the three-dimensional coordinates of the receiver and the receiver relative to the selected time reference system are obtained. The clock difference finally completes the receiver time, position, and speed calculations. When calculating the receiver motion velocity vector, it is necessary to construct the observation equation set as the solution position. But at this time, the observation equations are the differential form of the observational equations. When the distance change between the receiver and the satellite is changed to the carrier phase observation value, the carrier wave length is about $19 \mathrm{~cm}$, which can improve the accuracy of the measurement; the satellite velocity vector can be calculated as satellites [5]. The differential form of the position is obtained, and then the least squares method can be used to solve for the receiver positioning position.

\section{EXPERIMENTS AND ANALYSIS}

Figure 2 (a) and (b) respectively show the satellite space distribution and satellite signal-to-noise ratio in Beidou/GPS dual-mode environment. As can be seen from the figure, there are 22 satellites captured, of which 10 are GPS satellites and 12 are Beidou satellites. There are 17 satellites that are in good health and used for positioning, among which there are 9 GPS satellites and Beidou. There are 8 satellites; the signal-to-noise ratio of all satellites is above $40 \mathrm{~dB}$, and the signals are in good condition.

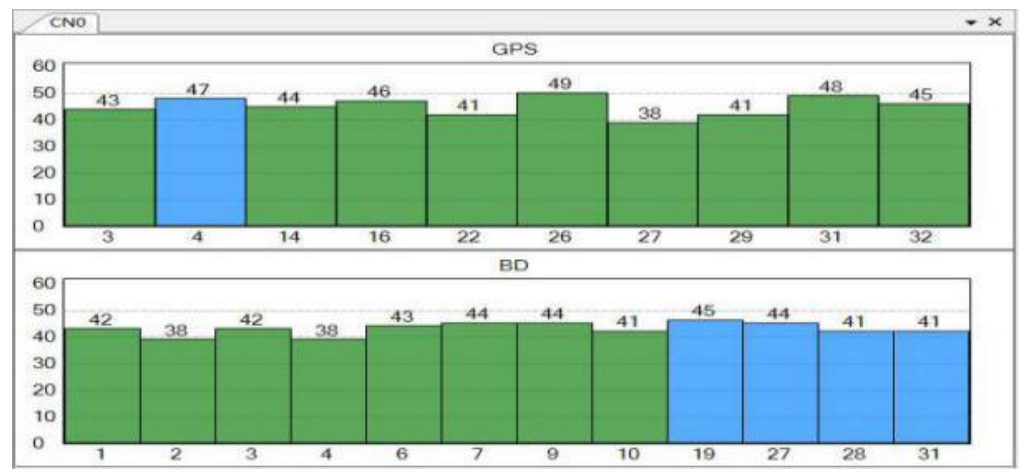

(a) Beidou/GPS satellite space constellation 


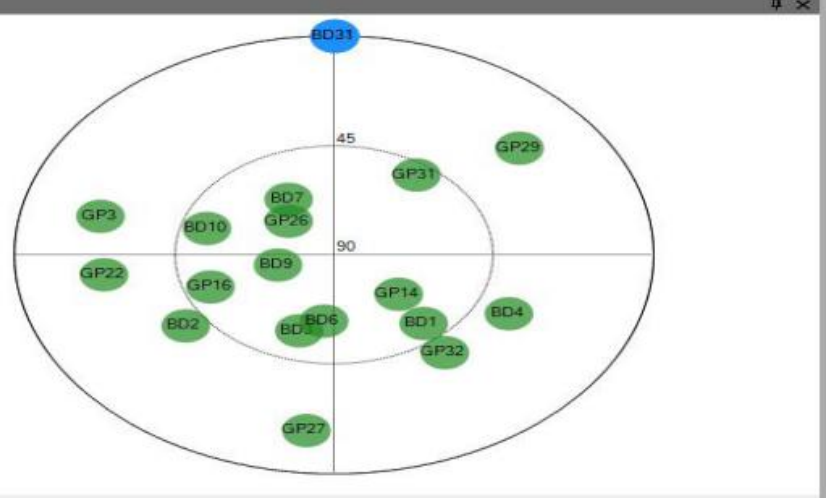

(b)Beidou/GPS satellite signal to noise ratio

FIG.2. Capture BeiDou/GPS satellite status

TAB.1. Beidou/GPS Dual Mode System Precision Factor

\begin{tabular}{cccc}
\hline PDOP & HDOP & VDOP & TDOP \\
\hline 1.40 & 1.30 & 0.40 & 0.00 \\
\hline
\end{tabular}

Figure 3 shows the positioning and speed results of the Beidou/GPS dual-mode system. The zero-error point (ie, the center of the circle) in Figure 3(a) is the actual position set by the software (through the measuring receiver). Long-term static positioning of the machine). It can be concluded that the $95 \%$ CEP positioning error of the Beidou/GPS dual-mode system is about $7 \mathrm{~m}$, and the speed measurement result is $0 \mathrm{~m} / \mathrm{s}$.

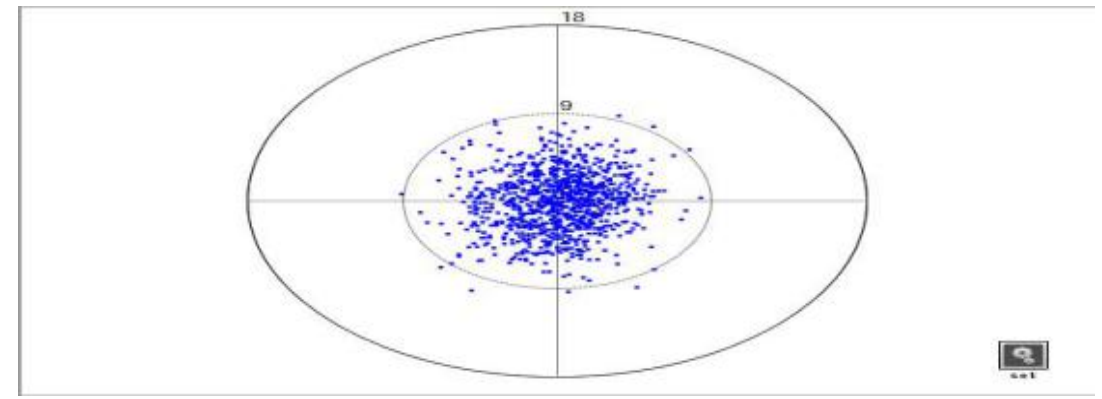

(a)Horizontal positioning results

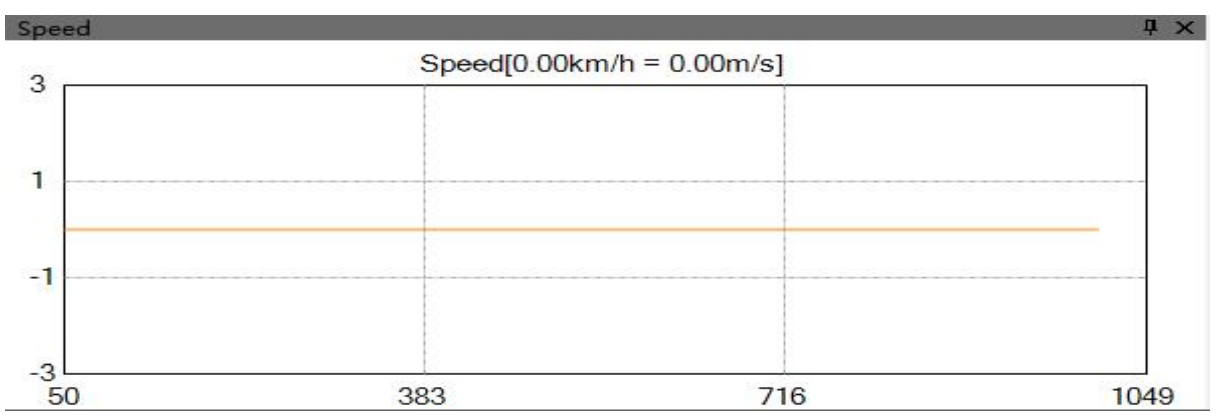

(b) Speed measurement result

FIG.3. Beidou/GPS dual-mode system positioning test chart 


\section{CONCLUSION}

This article describes the design and implementation of a GPS / BD dual-mode navigation receiver, through the FPGA + ARM architecture approach to satellite signal processing, and ultimately achieve navigation and positioning. Watson HX-BS282A converts the two RF signals received from the antenna to an IF signal. The sampled digital signal is processed by the FPGA to complete the baseband processing. The signal capture, tracking, and other functions are performed. Finally, the bus and the ARM are connected. Signal data navigation solution and positioning. From the experimental analysis, it can be seen that based on the Beidou/GPS dual-mode receiver, more observations are added on the basis of constellation optimization, which significantly reduces the positioning error and improves the positioning accuracy.

\section{ACKNOWLEDGMENTS}

Thepaperis supported by funds from Department of Science and Technology at Guangdong Province and Guangzhou City (No. 201604010051, 2015B090901060, 2016B090903001, 2016B090904001, 2016B090918126i1/4 (E2016KZ010101)

\section{REFERENCES}

1. Liu Weizhou, Wu Jicang. Analysis of Satellite Visibility and DOP Value in GPS/Beidou Integrated Navigation System [C]// China Satellite Navigation Academic Annual Conference on Electronics. 2012.

2. Barker B C, Betz J W, Clark J E, et al. Overview of the GPS M Code Signal [J]. Proceedings of the National Technical Meeting of the Institute of Navigation, 2000, 8218(1):1115-1128.

3. Li Fei. FPGA Implementation of Digitally Controlled Oscillator (NCO) [J]. Electronic Components Applications, 2010(11):42-44.

4. Kovár P, Kacmarik P, Vejrazka F. Interoperable GPS, GLONASS and Galileo software receiver [J]. IEEE Aerospace \& Electronics Systems Magazine, 2011, 26(4):24-30.

5. Kim T H, Sin C S, Lee S, et al. Analysis of performance of GPS L1 signal generator in GPS L1 signal[C]// International Conference on Control, Automation and Systems. IEEE, 2014:1006-1009. 